\title{
DOMINÂNCIA FISCAL OU DOMINÂNCIA MONETÁRIA NO BRASIL? UMA ANÁLISE DE CAUSALIDADE*
}

\author{
Sérgio Ricardo de Brito Gadelha ${ }^{\S}$ \\ José Angelo Divino
}

\section{RESUMO}

O objetivo deste estudo é verificar se existe dominância fiscal ou monetária na economia brasileira no período pós-Plano Real. Investiga-se a relação de equilíbrio de longo prazo e a causalidade de Granger bivariada e multivariada entre as variáveis taxa nominal de juros, relação dívida/PIB, relação superávit primário/PIB, taxa real de câmbio e o prêmio de risco. Os resultados sugerem que a economia brasileira encontra-se sob regime de dominância monetária, segundo as definições propostas por Sargent e Wallace (1981). Além disso, o modelo proposto por Blanchard (2004) não encontra apoio empírico no período analisado.

Palavras-chave: dominância fiscal, dominância monetária, causalidade de Engle-Granger.

\begin{abstract}
The aim of this study is to verify whether there is fiscal or monetary dominance in the Brazilian economy in the period of the post-Real plan. We investigate the long run equilibrium relationship and bivariate and multivariate Granger causality among the variables nominal interest rate, debt to GDP ratio, primary surplus to GDP ratio, real exchange rate and risk premium. The results have shown Brazil as a country under monetary dominance regime, according to Sargent and Wallace (1981) definition. In addition, the model proposed by Blanchard (2004) does not find empirical support in the Brazilian economy.
\end{abstract}

Keywords: fiscal dominance, monetary dominance, Engle-Granger Causality.

JEL classification: E63, H62.

* Os autores agradecem aos editores e a dois pareceristas anônimos por comentários e sugestões. As opiniões expressas nesse trabalho são de exclusiva responsabilidade dos autores, não expressando necessariamente a opinião da Secretaria do Tesouro Nacional.

\$ Mestre em Economia pela Universidade Católica de Brasília e Analista de Finanças e Controle da Secretaria do Tesouro Nacional. Endereço para contato: Secretaria do Tesouro Nacional, Coordenação Geral de Programação Financeira, Esplanada dos Ministérios, Bloco P, Anexo B, $1^{\circ}$ andar, sala 102 - Brasília - DF - CEP: 70048-900. E-mail: sergio.gadelha@fazenda.gov.br.

a Professor do Mestrado e Doutorado em Economia da Universidade Católica de Brasília. Endereço para contato: SGAN 916, sala A-116, Campus II - UCB - Brasília - DF - CEP: 70790-160. E-mail: jangelo@pos.ucb.br.

Recebido em setembro de 2007. Aceito para publicação em agosto de 2008. 


\section{INTRODUÇÃO}

As autoridades fiscal e monetária possuem instrumentos de política, objetivos e preferências diferentes, que podem levá-las a se comportarem de maneira antagônica e produzirem resultados indesejados. Por exemplo, taxas reais de juros elevadas podem ser usadas para estabilizar preços, mas podem também gerar uma trajetória explosiva da dívida pública. Em anos recentes, alguns estudos analisaram a interação entre essas duas autoridades sob a ótica da dominância fiscal ou monetária na economia brasileira, considerando três visões distintas.

A visão tradicional é apresentada por Sargent e Wallace (1981), em que o regime de dominância monetária é aquele em que a autoridade fiscal passiva gera um superávit primário compatível com a estabilização da relação dívida/PIB, de modo que a autoridade monetária ativa não é forçada a monetizar a dívida pública, mantendo o controle do nível de preços determinado pela demanda e oferta de moeda. No caso oposto, o regime de dominância fiscal é aquele em que a autoridade fiscal ativa gera superávit primário independente da necessidade de estabilização da relação dívida/PIB, e a autoridade monetária passiva perde o controle do nível de preços por ser forçada a gerar as receitas de senhoriagem necessárias à solvência do governo. E a inflação, ainda que motivada por desequilíbrios fiscais, é vista como um fenômeno monetário.

Sob essa visão, Tanner e Ramos (2002) examinaram questões referentes à solvência intertemporal e ajustamento fiscal no Brasil, usando dados fiscais mensais para o período 1991-2000. Os resultados indicaram um regime de dominância fiscal devido a um comportamento fiscal não sustentável durante o período, exceto entre 1995-1997. Ou seja, após a implantação do Plano Real e antes das crises asiáticas, evidenciou-se um regime de dominância monetária porque o governo buscou controlar o nível de endividamento.

A segunda visão fundamenta-se nas contribuições de Cochrane (2001), Sims (1994) e Woodford (1994, 1995, 2001), em que a Teoria Fiscal do Nível de Preços (TFNP) postula o papel da política fiscal na determinação do nível de preços. No regime fiscal Ricardiano (ou regime de dominância monetária), os superávits primários são gerados para assegurar a solvência fiscal para qualquer trajetória que o nível de preços possa tomar, e os preços são determinados segundo a visão tradicional. Por outro lado, no regime fiscal não-Ricardiano (regime de dominância fiscal), os superávits primários são gerados arbitrariamente, sem a preocupação de se manter a trajetória da dívida pública constante. O nível de preços é ajustado para satisfazer o valor presente da restrição orçamentária.

Utilizando dados mensais do período 1995-2003, Fialho e Portugal (2005) verificaram as hipóteses das dominâncias segundo a TFNP, modelando uma relação entre as séries de dívida/ PIB e superávit primário/PIB por meio de um VAR e analisando as funções de impulso-resposta. Os resultados indicaram um regime de dominância monetária devido à resposta da dívida pública a inovações no superávit primário. 
A terceira visão é baseada no estudo de Blanchard (2004), que elaborou um modelo estrutural em que a política monetária restritiva provoca dinâmicas explosivas da dívida pública sob um regime de metas de inflação e buscou na evidência empírica brasileira de 2002 e 2003 a corroboração de seus argumentos. Afirma que um aumento da taxa nominal de juros em resposta ao aumento da inflação acima da meta estipulada aumentou não só o estoque da dívida pública para além do seu limite sustentável, por meio do impacto sobre o serviço dessa dívida, mas também a probabilidade de default e os prêmios de riscos, levando a uma fuga de capitais externos e a uma depreciação do real, ao invés de uma apreciação. Como uma grande parcela da dívida pública estava indexada ao dólar, a desvalorização cambial provocou um aumento da dívida pública e afetou as expectativas de inflação, desencadeando um processo inflacionário e criando, então, um círculo vicioso. A instabilidade econômica provocada pela incerteza eleitoral de 2002 é considerada por Blanchard (2004) um exemplo de como a trajetória de variáveis fiscais pode afetar a condução da política monetária, caracterizando um regime de dominância fiscal. Vale ressaltar, porém, que esse modelo possui algumas limitações. Especificamente, é um modelo estático e não considera o canal de transmissão da política monetária sobre a demanda agregada.

Observa-se, assim, que a questão da dominância fiscal ou monetária é um tema não consensual no atual debate acadêmico, com importantes implicações de política econômica. $\mathrm{O}$ objetivo principal deste trabalho é utilizar análises de causalidade bivariada e multivariada para verificar sob qual regime de dominância encontra-se a economia brasileira no período do pósplano Real. A análise de causalidade revela-se adequada nesse contexto, porque permite testar a definição proposta por Sargent e Wallace (1981), segundo a qual a existência de causalidade unidirecional do superávit primário para a dívida pública é consistente com um regime de dominância monetária, ao passo que uma causalidade unidirecional em sentido oposto, da dívida pública para o superávit primário, define um regime de dominância fiscal. Além disso, o teste de causalidade permite verificar se há transmissão entre variáveis fiscais e monetárias conforme assumido a priori por Blanchard (2004) para argumentar que a economia brasileira encontravase sob dominância fiscal.

Os resultados da análise de causalidade, tanto bivariada quanto multivariada, indicam que o Brasil encontra-se sob dominância monetária. Há uma causalidade unidirecional do superávit primário para a dívida pública, caracterizando um regime de dominância monetária. Este resultado é robusto às versões bivariada e multivariada do teste de causalidade para variáveis co-integradas. Além disso, a transmissão entre variáveis fiscais e monetárias assumida por Blanchard (2004) não encontra respaldo empírico na análise de causalidade.

O artigo está organizado da seguinte forma: a próxima seção apresenta as variáveis envolvidas na análise; a terceira seção descreve o tratamento econométrico, onde são apresentados os testes de raiz unitária e de causalidade multivariado e bivariado; a quarta seção reporta e analisa os resultados; finalmente, a quinta seção conclui o artigo.

1 Agradecemos a um parecerista anônimo por ter chamado nossa atenção para essas limitações do modelo de Blanchard (2004). 


\section{DADOS}

Com base nos modelos de Blanchard (2004) e de Sargent e Wallace (1981), as variáveis envolvidas na análise de causalidade são: taxa de juros, dívida pública, superávit primário, taxa de câmbio real e risco país. A análise foi realizada para o período pós-Plano Real, quando a economia brasileira atingiu estabilidade de preços por meio do papel ativo assumido pela política monetária. Assim, os dados consistem de observações mensais para o período de janeiro de 1995 a dezembro de 2005.

A taxa de juros nominal, $i$, foi representada pelo over selic, que é o instrumento de política monetária do Banco Central do Brasil. A relação dívida/PIB, $d_{t}$, constitui a dívida líquida do setor público, compreendendo o governo federal e Banco Central do Brasil, governos estaduais, governos municipais e empresas estatais (federais, estaduais e municipais), como porcentagem do Produto Interno Bruto (PIB). A relação superávit primário/PIB, $s p_{t}$, são as necessidades de financiamento do setor público, formado por governo central (governo federal mais o Banco Central), governos estaduais, governos municipais e empresas estatais (federais, estaduais e municipais), em porcentagem do PIB, com desvalorização cambial sobre estoque da dívida mobiliária interna. A taxa real de câmbio, $z_{t}$, é o preço de um dólar americano expresso em reais deflacionado pelo diferencial de preços. Isto é, $z_{t}=e_{t}\left(P_{t}^{*} / P_{t}\right)$, onde $z_{t}$ é a taxa real de câmbio, $e_{t}$ é a taxa nominal de câmbio (R\$/US\$), $P_{t}^{*}$ é o Índice de Preços ao Consumidor dos Estados Unidos e $P_{t}$ é o IPCA brasileiro. O risco país é representado pelo Spread do Emerging Markets Bond Index $\left(e m b i_{t}\right)$. O embi mede a diferença entre o rendimento de um título expresso em dólar emitido pelo governo brasileiro e um título correspondente emitido pelo Tesouro dos Estados Unidos da América. Será utilizado como proxy para o prêmio de risco.

As variáveis foram tomadas em forma logarítmica. Com exceção do embi, cuja origem foi o JP Morgan, todas as demais variáveis foram obtidas no sítio eletrônico do Instituto de Pesquisa Econômica e Aplicada. ${ }^{2}$

\section{TRATAMENTO ECONOMÉTRICO}

\subsection{Testes de raízes unitárias}

Os testes modificados de Dickey-Fuller $\left(\mathrm{MADF}^{\mathrm{GLS}}\right)$ e de Phillips-Perron (MPP $\left.{ }^{\mathrm{GLS}}\right)$, propostos por Elliot, Rottemberg e Stock (1996), e Ng e Perron (2001) são aplicados para verificar a estacionariedade das séries porque superam os problemas de baixo poder estatístico e distorções de tamanho dos testes tradicionais de Dickey-Fuller (1979, 1981), Said e Dickey (1984) e de Phillips-Perron (1988).

2 Os dados estão disponíveis em: www.ipeadata.gov.br. 
As modificações no teste padrão de raiz unitária de Dickey e Fuller $(1979,1981)$ e de Said e Dickey (1984) fundamentam-se em dois aspectos centrais: (a) a extração de tendência em séries de tempo usando mínimos quadrados ordinários (OLS) é ineficiente, e (b) a importância de uma seleção apropriada para a ordem de defasagem do termo aumentado, de modo a obter uma melhor aproximação para o verdadeiro processo gerador de dados.

No primeiro caso, (a), Elliot, Rottemberg e Stock (1996) propõem usar mínimos quadrados generalizados (GLS) para extrair a tendência estocástica da série. Emprega-se o procedimento padrão para estimar a estatística $\mathrm{ADF}^{\mathrm{GLS}}$ como sendo a estatística $t$ para testar a hipótese nula $H_{0}: \beta_{0}=0$, indicando a presença de raiz unitária, da seguinte regressão estimada por mínimos quadrados ordinários:

$$
\Delta \tilde{y}_{t}=\beta_{0} \tilde{y}_{t-1}+\sum_{j=1}^{k} \beta_{j} \Delta \tilde{y}_{t-j}+e_{t k}
$$

contra a hipótese alternativa $H_{A}: \beta_{0}<0$, de que a série é estacionária. Em (1), $\tilde{y}_{t}$ é a série com tendência removida por mínimos quadrados generalizados, $\Delta$ é o operador de primeiras diferenças, $e_{t k}$ é o resíduo não autocorrelacionado e homocedástico.

Com relação ao segundo aspecto, (b), Ng e Perron (2001) demonstram que os critérios de informação de Akaike (AIC) e de Schwarz (SIC) tendem a selecionar baixos valores para a defasagem $k$, quando se tem uma grande raiz negativa (próximo a -1) no polinômio de médias móveis da série, conduzindo os testes de raízes unitárias a sérias distorções. Isso motivou o desenvolvimento do critério modificado de informação de Akaike (MAIC) para a seleção da defasagem auto-regressiva, de modo a minimizar as distorções provocadas por seleção inadequada de defasagem na equação (1).

Ng e Perron (2001) propõem que as mesmas modificações sejam aplicadas também ao teste tradicional de Phillips e Perron (1988), originando o teste MPP ${ }^{\mathrm{GLS}}$. Por meio de simulações, Ng e Perron (2001) mostram que a aplicação conjunta de GLS para extrair a tendência determinista e do critério de seleção de defasagens MAIC produzem testes com maior poder e menores distorções de tamanho estatístico quando comparados aos testes tradicionais ADF e PP.

Contudo, mesmo os testes modificados, possuem baixo poder na presença de quebras estruturais, tornando-se viesados no sentido da não-rejeição da hipótese nula de existência de raiz unitária quando a série é estacionária. Perron (1989) propõe alterações nos testes de raízes unitárias a fim de contornar essa dificuldade. A quebra estrutural é tratada como um evento exógeno, conhecendo-se sua data de ocorrência. Seja $\tau$ o período anterior à quebra estrutural, de modo que a hipótese nula é que a série $y_{t}$ segue um processo de raiz unitária com quebra estrutural no período $t=\tau+1$, contra a hipótese alternativa de que $y_{t}$ é estacionária. Em sua 
forma geral, denominado modelo (C), considera quebra de intercepto e de tendência e é expresso por:

$$
y_{t}=a_{0}+a_{1} y_{t-1}+a_{2} t+\mu_{1} D_{L}+\mu_{2} D_{p}+\mu_{3} D_{T}+\sum_{i=1}^{k} \beta_{i} \Delta y_{t-i}+\varepsilon_{t}
$$

onde $D_{P}=1$ se $t=\tau+1$ e zero caso contrário, $D_{L}=1$ se $t>\tau$ e zero caso contrário, $D_{T}=t-\tau$ se $t>\tau$ e zero caso contrário, $\varepsilon_{t}$ é o resíduo não autocorrelacionado e homocedástico.

Os resíduos obtidos em (2) são usados para estimar a seguinte equação via OLS:

$$
\hat{\varepsilon}_{t}=a_{1} \hat{\varepsilon}_{t-1}+v_{t}
$$

Sob a hipótese nula de raiz unitária, o valor teórico de $a_{1}$ é unitário. Se os resíduos são independentes e identicamente distribuídos, a distribuição de $a_{1}$ dependerá da fração de quebra, denotada por $\lambda=\tau / T$, onde $T$ é o número total de observações. Caso os resíduos sejam correlacionados, a equação (3) deve assumir a forma do teste ADF, com seleção apropriada de defasagens para corrigir a autocorrelação. A estatística $t$ é calculada para a hipótese nula de que $a_{1}=1$ pode ser comparada com os valores críticos tabulados por Perron (1989).

\subsection{Causalidade multivariada}

A análise de causalidade inicia-se pela estimação de um modelo vetorial auto-regressivo (VAR), envolvendo todas as variáveis mencionadas anteriormente. O conceito de causalidade no sentido de Granger está associado à idéia de precedência temporal entre variáveis. Assim, se $y_{t}$ contém informação passada que ajuda na previsão de $z_{t}$, e se essa informação não está contida em outras séries usadas no modelo, então $y_{t}$ Granger-causa $z_{t}$ [Granger (1969)]. O VAR em forma reduzida de dimensão $p$ pode ser escrito com:

$$
X_{t}=A_{0}+A_{1} X_{t-1}+A_{2} X_{t-2}+\ldots+A_{p} X_{t-p}+\zeta_{t}
$$

onde, $X_{t}$ é um vetor de variáveis estacionárias, $p$ é o número de defasagens, $A_{0}$ é um vetor de interceptos, $A_{i}$ são matrizes de coeficientes e $\zeta_{t}$ é um vetor de resíduos não-autocorrelacionados e homocedásticos. No caso, o vetor $X_{t}$ contém as séries de taxa de juros, taxa real de câmbio, EMBI, relação dívida/PIB e relação superávit/PIB. A seleção da ordem de defasagem, $p$, é feita pelos critérios de informação de Akaike e Scharwz. Como a análise de causalidade não requer a estimação de parâmetros estruturais, não há necessidade de se adotar alguma estratégia de identificação em (4).

3 A hipótese nula impõe as seguintes restrições nos parâmetros da equação (2): $a_{1}=1, \mu_{1} \neq 0, \mu_{2} \neq 0$ e $a_{2}=\mu_{3}=0$, ao passo que a hipótese alternativa, tem-se: $\left|a_{1}\right|<1, a_{2} \neq 0, \mu_{2} \neq 0, \mu_{3} \neq 0$ e $\mu_{1}=0$. Perron (1989) prevê, ainda, dois casos particulares de mudanças no intercepto ou na inclinação da série. 
A causalidade de Granger da variável $j$ para a variável $h$ é avaliada testando a hipótese nula de que os coeficientes da variável $j$ em todas as suas defasagens são, simultaneamente, estatisticamente iguais a zero na equação em que $h$ é a variável dependente. Caso a hipótese nula seja rejeitada, conclui-se que a variável $j$ Granger-causa a variável $h$.

Caso as variáveis em $X_{t}$ não sejam estacionárias, deve-se testar co-integração. Nesse caso, havendo co-integração entre as variáveis, utiliza-se o vetor co-integrante para obter uma combinação linear estacionária entre as variáveis e estima-se um modelo vetorial auto-regressivo de correção de erros, chamado VEC. A significância estatística do termo de correção de erros também deve ser considerada na condução do teste de causalidade.

A co-integração pode ser testada seguindo o procedimento proposto por Johansen e Juselius (1990) e Johansen (1991). O teste consiste em modelar séries temporais como uma regressão de posto reduzido em que se calculam as estimativas de máxima verossimilhança do vetor cointegrante no modelo de correção de erros multivariado com erros gaussianos. O teste requer a estimação da representação de correção de erros dada por:

$$
\Delta X_{t}=\mu+\pi X_{t-1}+\sum_{i=1}^{p-1} \pi_{i} \Delta X_{t-i}+\varepsilon_{t}
$$

onde $X_{t}$ é um vetor coluna $(5 \times 1)$ de variáveis, $\mu$ é um vetor $(5 \times 1)$ de termos constantes, $\pi$ e $\pi_{i}$ representam matrizes de coeficientes, $p$ é a ordem das defasagens e o resíduo $\varepsilon_{t}$ é não autocorrelacionado e homocedástico. A matriz coeficientes $\pi$ é conhecida como matriz co-integrante e contém informações sobre o equilíbrio de longo prazo entre as variáveis. O número de autovalores de $\pi$ que são estatisticamente diferentes de zero, o que corresponde ao posto de $\pi$, fornece o número de vetores co-integrantes no sistema. Johansen propõe as estatísticas do traço e do autovalor máximo para se testar quantos vetores co-integrantes existem em (5). Essas estatísticas são:

$$
\begin{aligned}
& \lambda_{\text {traco }}(r)=-T \sum_{i=r+1}^{n} \ln \left(1-\hat{\lambda}_{i}\right) \\
& \lambda_{\text {max }}(r, r+1)=-T \ln \left(1-\hat{\lambda}_{r+1}\right)
\end{aligned}
$$

onde $\hat{\lambda}_{i}$ são os valores estimados dos autovalores obtidos da matriz $\pi$ e $T$ é o número de observações. O teste segue um procedimento recursivo, onde a hipótese nula é de que há, no máximo, $r$ vetores co-integrantes. Valores críticos foram tabulados por Johansen e Juselius (1990) e Johansen (1991). 


\subsection{Causalidade bivariada}

A análise bivariada de causalidade no sentido de Granger envolve a estimação de modelos auto-regressivos de defasagens distribuídas (ADL) para pares de variáveis. A modelagem ADL é abrangente e permite capturar a dinâmica do sistema, sem incorrer no viés de omissão de defasagens relevantes. Além disso, não impõe a restrição de que todas as equações tenham o mesmo número de defasagens em cada variável, como ocorre na estimação de VAR. Contudo, causalidade bivariada pode ser afetada por omissão de variável no sistema. Essa limitação foi superada pelo teste multivariado de causalidade de Granger, descrito na seção 3.2.

O teorema da representação de Granger postula que se as séries de tempo $y_{t}$ e $z_{t}$ são cointegradas, então a regressão entre elas pode ser expressa com um modelo de correção de erros (ENGLE; GRANGER, 1987). Logo, quando as variáveis são co-integradas, a causalidade de Granger deve ser investigada inserindo-se o resíduo defasado de um período da regressão cointegrante, chamado mecanismo de correção de erros, nos modelos auto-regressivos e de defasagens distribuídas (ADL) em primeiras diferenças. Isto é,

$$
\begin{aligned}
& \Delta y_{t}=\alpha_{1}+\alpha_{y} \hat{e}_{y z, t-1}+\sum_{i=1}^{p} \alpha_{11}(i) \Delta y_{t-i}+\sum_{i=1}^{q} \alpha_{12}(i) \Delta z_{t-i}+\varepsilon_{y t} \\
& \Delta z_{t}=\alpha_{2}+\alpha_{z} \hat{e}_{z y, t-1}+\sum_{i=1}^{l} \alpha_{21}(i) \Delta y_{t-i}+\sum_{i=1}^{m} \alpha_{22}(i) \Delta z_{t-i}+\varepsilon_{z t}
\end{aligned}
$$

onde $\varepsilon_{y t}$ e $\varepsilon_{z t}$ são resíduos não autocorrelacionados, $\hat{e}_{y z, t-1}=\left(y_{t-1}-\beta z_{t-1}\right)$ e $\hat{e}_{z y, t-1}=\left(z_{t-1}-\varphi y_{t-1}\right)$ são os mecanismos de correção de erros (MCE).

Nas equações (8) e (9), a hipótese nula $H_{0}: \alpha_{12}(i)=0$ e $\quad \alpha_{y}=0$ significa que $\Delta z_{t}$ não Granger-causa $\Delta y_{t}$, ao passo que a hipótese alternativa $H_{A}: \alpha_{12}(i) \neq 0$ e $\alpha_{y} \neq 0$ significa que $\Delta z_{t}$ Granger-causa $\Delta y_{t}$. Alternativamente, a hipótese nula $H_{0}: \alpha_{21}(i)=0$ e $\alpha_{z}=0$ significa que $\Delta y_{t}$ não Granger-causa $\Delta z_{t}$, ao passo que a hipótese alternativa $H_{A}: \alpha_{21}(i) \neq 0$ e $\alpha_{z} \neq 0$ significa que $\Delta y_{t}$ Granger-causa $\Delta z_{t}$. Com base nessas hipóteses, são possíveis 4 resultados para cada par de variáveis.

O teste de causalidade de Granger é sensível ao número de defasagens incluídas nos modelos ADL, influenciando na direção da causalidade. Por esse motivo, foram utilizados critérios rigorosos de seleção de defasagens, baseados nos critérios AIC e SIC e na abordagem "geral para o específico" indicada por Campbell e Perron (1991).

4 Nessa abordagem, escolhe-se a priori um número máximo de defasagens $\left(\mathrm{p}_{\text {máximo }}\right)$, as quais vão sendo eliminadas uma a uma caso o coeficiente da última defasagem se apresente não-significativo. A defasagem ótima $(p)$ será determinada quando o teste $t$ do coeficiente estimado exceder o valor de 1,68, em termos absolutos, ao nível de significância de $10 \%$, caso em que $\mathrm{p}=\mathrm{p}_{\text {ótimo }}$. 


\section{Resultados}

\subsection{Testes de raízes unitárias}

Os resultados dos testes modificados de Dickey-Fuller aumentado (MADF ${ }^{\mathrm{GLS}}$ ) e de Phillips-Perron $\left(\mathrm{MPP}^{\mathrm{GLS}}\right)$, apresentados na Tabela 1, indicam que as séries são integradas de primeira ordem. A hipótese nula de raiz unitária não foi rejeitada para todas as variáveis em nível. Em primeira diferença, por outro lado, as séries se mostraram estacionárias.

A Tabela 2 apresenta os resultados do teste de Perron (1989). A data da primeira quebra estrutural considerada foi o mês de fevereiro de 1999 devido à mudança de regime cambial na economia brasileira, ${ }^{5}$ obtendo-se uma fração de quebra $\lambda=0,4$, aproximadamente. A data da segunda quebra estrutural foi o mês de abril de 2002, período em que a taxa real de câmbio e o embi apresentam tendência de aumento devido à crise de confiança externa sobre a evolução da economia brasileira no período pré-eleitoral. Nesse caso, obteve-se uma fração de quebra $\lambda=0,7$, onde $\tau$ corresponde ao período de janeiro de 1995 a março de 2002.

\section{Tabela 1 - Testes de raiz unitária}

\begin{tabular}{|c|c|c|c|c|c|}
\hline Variável & Modelo & MADF $^{\text {GLS }}$ & $\mathrm{MPP}^{\mathrm{GLS}}$ & lags & Resultado \\
\hline$i_{t}$ & C & $-0,86$ & $-0,86$ & 1 & $\mathrm{I}(1)$ \\
\hline$i_{t}$ & $C, T$ & $-2,40$ & $-2,36$ & 1 & $\mathrm{I}(1)$ \\
\hline$z_{t}$ & C & $-0,59$ & $-0,61$ & 2 & $\mid(1)$ \\
\hline$z_{t}$ & $\mathrm{C}, \mathrm{T}$ & $-1,08$ & $-1,10$ & 2 & $\mathrm{I}(1)$ \\
\hline$e m b i_{t}$ & C & $-1,47$ & $-1,56$ & 1 & $\mathrm{I}(1)$ \\
\hline$e m b i_{t}$ & $C, T$ & $-2,21$ & $-2,26$ & 1 & $\mathrm{I}(1)$ \\
\hline$d_{t}$ & C & 0,13 & 0,19 & - & $\mathrm{I}(1)$ \\
\hline$d_{t}$ & $\mathrm{C}, \mathrm{T}$ & $-1,01$ & $-1,00$ & - & $\mathrm{I}(1)$ \\
\hline$s p_{t}$ & C & $-1,35$ & $-1,37$ & 2 & $\mid(1)$ \\
\hline$s p_{t}$ & $\mathrm{C}, \mathrm{T}$ & $-1,54$ & $-1,53$ & 2 & I(1) \\
\hline
\end{tabular}

Nota: "C" significa constante. "T" significa tendência determinística. $\left({ }^{*}\right)$ significância a $1 \%$; $(* *)$ significância a $5 \%$; $(* *)$ significância a 10\%. O número ótimo de defasagens segue MAIC, começando com 14 defasagens. Os valores críticos do teste $\mathrm{MADF}^{\mathrm{GLS}}$ são: (i) modelo com constante: -2,58 (1\%); -1,94 (5\%); e -1,61 (10\%); (ii) modelo com constante e tendência: $-3,54(1 \%) ;-3,00(5 \%) ;-2,71(10 \%)$. Os valores críticos do teste MPP ${ }^{\text {GLS }}$ são: (i) modelo com constante: $-2,58$ (1\%); -1,98(5\%); e -1,62 (10\%); (ii) modelo com constante e tendência: -3,42 (1\%); -2,91 (5\%); -2,62 (10\%)

5 Além da mudança do regime de câmbio fixo para o regime de câmbio flutuante, o ano de 1999 também foi marcado pela implantação do regime de metas de inflação e início de rigoroso ajuste fiscal das contas públicas. 
Tabela 2 - Teste de Perron para raiz unitária na presença de quebra estrutural ${ }^{6}$

\begin{tabular}{lccccc}
\hline \multirow{2}{*}{ Variáveis } & \multicolumn{2}{c}{$1^{\mathrm{a}}$ quebra: $1999: 02$} & \multicolumn{2}{c}{$2^{\mathrm{a}}$ quebra: 2002:4 } & \multirow{2}{*}{ Resultado } \\
\cline { 2 - 5 } & Modelo & $\mathrm{t}$-Perron & Modelo & t-Perron & \\
\hline$z_{t}$ & Modelo A & $-1,36$ & Modelo B & $-3,34$ & $\mathrm{I}(1)$ \\
$i_{t}$ & Modelo B & $-3,27$ & Modelo B & $-3,28$ & $\mathrm{I}(1)$ \\
$e m b i_{t}$ & Modelo B & $-2,40$ & Modelo B & $-3,16$ & $\mathrm{I}(1)$ \\
$d_{t}$ & Modelo B & $-2,51$ & Modelo B & $-3,22$ & $\mathrm{I}(1)$ \\
$s p_{t}$ & Modelo B & $-3,27$ & Modelo B & $-3,59$ & $\mathrm{I}(1)$ \\
\hline
\end{tabular}

Nota: Para $\lambda=0,4$, os valores críticos do modelo (A) são: -4,34 (1\%), -3,72 (5\%), -3,44 (10\%), e do modelo (B) são: -4,55 (1\%), $-3,94(5 \%)$ e $-3,66(10 \%)$. Para $\lambda=0,7$, os valores críticos do modelo (B) são: $-4,51(1 \%),-3,85(5 \%)$ e $-3,57(10 \%)$.

Em ambos os casos, o teste de Perron (1989) confirma os resultados dos testes MADF ${ }^{\text {GLS }}$ e MPP $^{\mathrm{GLS}}$, indicando que as séries possuem raiz unitária. Observa-se, assim, de acordo com os resultados das Tabelas 1 e 2, que todas as variáveis representam processos integrados de primeira ordem, ou $\mathrm{I}(1)$.

\subsection{Causalidade multivariada}

Os resultados do teste de co-integração de Johansen são reportados na Tabela 3. Foram utilizadas duas defasagens no VAR estimado, selecionadas pelos critérios de informação de Akaike e Schwarz. Os valores calculados das estatísticas $\lambda_{\text {traço }}$ e $\lambda_{\max }$ indicam que as variáveis no sistema são co-integradas e que há apenas um vetor co-integrante. Esse resultado indica que a análise de causalidade multivariada deve ser conduzida sob a estimação de um VEC, conforme descrito na seção 3.2 .

Tabela 3 - Resultados do teste de co-integração de Johansen

\begin{tabular}{ccccc}
\hline rank $(r)$ co-integrante & $\lambda_{\text {traco }}$ & Prob. & $\lambda_{\text {max }}$ & Prob. \\
\hline$r=0$ & $79,64^{\star \star}$ & 0,0309 & $32,39^{\star \star \star}$ & 0,0946 \\
$r \leq 1$ & 47,25 & 0,1764 & 21,78 & 0,2885 \\
$r \leq 2$ & 25,47 & 0,3723 & 12,95 & 0,5619 \\
$r \leq 3$ & 12,52 & 0,4033 & 8,67 & 0,4700 \\
$r \leq 4$ & 3,85 & 0,4347 & 3,85 & 0,4347 \\
\hline
\end{tabular}

Nota: ** Significa rejeição da hipótese nula de posto $r$ co-integrante ao nível de significância de $5 \% .{ }^{* *}$ Indica rejeição da hipótese nula de posto $r$ co-integrante ao nível de significância de 10\%. "Prob." significa valor-p.

6 No modelo (A), a hipótese nula impõe as seguintes restrições aos parâmetros da equação (2): $a_{1}=1, a_{2}=\mu_{1}=\mu_{3}=0$ e $\mu_{2} \neq 0$, ao passo que, na hipótese alternativa, consideram-se as seguintes restrições: $\left|a_{1}\right|<1, a_{2} \neq 0, \mu_{1} \neq 0, \mu_{2}=\mu_{3}=0$. Já no modelo (B), a hipótese nula é construída impondo-se as seguintes restrições nos parâmetros da equação (1): $a_{1}=1, \mu_{2}=\mu_{3}=a_{2}=0$ e $\mu_{1} \neq 0$, enquanto a hipótese alternativa requer: $a_{1}<1, a_{2} \neq 0, \mu_{3} \neq 0, \mu_{1}=0$. 
A Tabela 4 apresenta os resultados do teste de causalidade de Granger baseado na estimação de um VEC. Observe que a relação superávit primário/PIB Granger-causa unidirecionalmente a relação dívida/PIB ao nível de significância de 5\%, mostrando que a geração de superávits primários tem conseguido controlar a dinâmica da dívida pública. Esse resultado indica ganhos de credibilidade da política fiscal e caracteriza um regime de dominância monetária.

Além disso, a taxa Selic Granger-causa unidirecionalmente a relação dívida/PIB ao nível de significância de $10 \%$, demonstrando que a dinâmica da dívida pública não interfere na postura assumida pela política monetária, demonstrando ganhos de credibilidade da política monetária.

Esses dois resultados indicam a existência de um regime de dominância monetária, segundo a definição proposta por Sargent e Wallace (1981).

Tabela 4 - Resultados do teste de causalidade de Granger aplicado ao VEC

\begin{tabular}{|c|c|c|c|c|c|}
\hline \multirow{2}{*}{$\begin{array}{l}\text { Variáveis } \\
\text { Indepedentes }\end{array}$} & \multicolumn{5}{|c|}{ Variáveis Dependentes } \\
\hline & $\Delta d_{t}$ & $\Delta e m b i_{t}$ & $\Delta z_{t}$ & $\Delta i_{t}$ & $\Delta s p_{t}$ \\
\hline$\Delta d_{t}$ & - & $\begin{array}{c}3,102698^{\star \star \star} \\
{[0,0782]}\end{array}$ & $\begin{array}{c}49,47143^{\star} \\
{[0,0000]}\end{array}$ & $\begin{array}{l}0,203406 \\
{[0,6520]}\end{array}$ & $\begin{array}{l}0,424240 \\
{[0,5148]}\end{array}$ \\
\hline$\Delta e m b i_{t}$ & $\begin{array}{c}19,66129^{\star} \\
{[0,0000]}\end{array}$ & - & $\begin{array}{c}8,822158^{*} \\
{[0,0030]}\end{array}$ & $\begin{array}{c}6,003751^{\star *} \\
{[0,0143]}\end{array}$ & $\begin{array}{l}0,996850 \\
{[0,3181]}\end{array}$ \\
\hline$\Delta z_{t}$ & $\begin{array}{c}11,06113^{\star} \\
{[0,0009]}\end{array}$ & $\begin{array}{c}2,740116^{\star \star \star} \\
{[0,0979]}\end{array}$ & - & $\begin{array}{l}0,145652 \\
{[0,7027]}\end{array}$ & $\begin{array}{l}0,802216 \\
{[0,3704]}\end{array}$ \\
\hline$\Delta i_{t}$ & $\begin{array}{c}3,490316^{\star \star *} \\
{[0,0617]}\end{array}$ & $\begin{array}{c}9,477634^{*} \\
{[0,0021]}\end{array}$ & $\begin{array}{c}7,061007^{\star} \\
{[0,0079]}\end{array}$ & - & $\begin{array}{l}0,022832 \\
{[0,8799]}\end{array}$ \\
\hline$\Delta s p_{t}$ & $\begin{array}{c}4,737692^{* *} \\
{[0,0295]}\end{array}$ & $\begin{array}{c}4,108312^{\star *} \\
{[0,0427]}\end{array}$ & $\begin{array}{l}1,510359 \\
{[0,2191]}\end{array}$ & $\begin{array}{c}4,530668^{\star *} \\
{[0,0333]}\end{array}$ & - \\
\hline
\end{tabular}

Nota: Os valores em colchetes são os valores-p. Todos os outros valores são testes $\mathrm{F}$ da Causalidade de Granger. $\left(^{*}\right)$ significância a 1\%, $\left(^{* *}\right)$ significância a 5\%, $\left(^{* *}\right)$ significância a 10\%. Selecionou-se uma defasagem ótima selecionada no VAR pelos critérios de informação de Akaike, Schwarz e de Hannan-Quinn. O modelo VEC foi estimado com a inclusão de variável dummy para fevereiro de 1999.

Observam-se outras relações de causalidade unidirecional. A relação superávit primário/ PIB Granger-causa o spread do EMBI e a taxa Selic ao nível de significância de 5\%. Isto mostra que há ganhos de credibilidade da política fiscal, a qual também não está condicionada a mudanças na percepção de riscos dos agentes internacionais. A taxa Selic Granger-causa a taxa real de câmbio ao nível de significância de $1 \%$, o que permite inferir que o Banco Central pode afetar a volatilidade da taxa de câmbio por meio do instrumento de política monetária. Antes do sistema de metas de inflação, adotado em 1999, o Banco Central praticava um regime de flutuação controlada para a taxa nominal de câmbio, afetando, assim, diretamente a volatilidade 
da taxa real de câmbio. Após a implantação do sistema de metas de inflação, o regime cambial torna-se de flutuação livre e o objetivo da política monetária passa a ser manter a inflação próxima à meta estabelecida. O efeito dessa política sobre a taxa de câmbio é indireto, determinado pelo diferencial entre o juro doméstico e o juro internacional. Aumentos nesse diferencial, por exemplo, devido a uma política monetária restritiva, tendem a atrair capital estrangeiro e a valorizar a taxa de câmbio. Esses resultados contradizem Blanchard (2004) que, implicitamente, assumiu uma causalidade unidirecional em sentido inverso entre as variáveis mencionadas anteriormente.

As relações de bicausalidade também contrariam a transmissão unidirecional assumida por Blanchard (2004). A existência de bicausalidade entre a relação dívida/PIB e o spread do EMBI mostra que o grau de endividamento público afeta a avaliação externa da economia brasileira. Por outro lado, o risco país tem importância para se entender as variações no grau de endividamento público devido ao efeito que exerce sobre a avaliação externa da economia brasileira.

A bicausalidade no sentido de Granger entre taxa Selic e o spread do EMBI revela que melhorias na avaliação externa da economia brasileira podem ser obtidas por meio da credibilidade da política monetária. Por outro lado, pode-se argumentar que a taxa Selic é elevada porque o País tem um risco maior. Observa-se, também, bicausalidade entre a relação dívida/PIB e a taxa real de câmbio, ao nível de significância de 1\%, e entre o spread do EMBI e a taxa real de câmbio.

Assim, a análise de causalidade multivariada permite concluir que o ciclo vicioso assumido por Blanchard (2004) e que levaria o País a uma situação de dominância fiscal não encontra evidência empírica favorável. Por outro lado, a causalidade unidirecional entre superávit primário/PIB e dívida/PIB e entre taxa de juros Selic e dívida/PIB são coerentes com a definição proposta por Sargent e Wallace (1981), indicando que o País encontra-se sob um regime de dominância monetária. A análise bivariada da próxima seção reforça esta conclusão.

\subsection{Causalidade bivariada}

Pelo teorema da representação de Granger, se as séries de tempo $y_{t}$ e $z_{t}$ são co-integradas, então a regressão entre elas pode ser expressa com um modelo de correção de erros (ENGLE; GRANGER, 1987). Como as variáveis envolvidas no teste de causalidade são I(1), a possibilidade de co-integração entre os pares de variáveis é investigada inserindo-se o mecanismo de correção de erros nos modelos ADL em primeiras diferenças. Este procedimento é equivalente a testar a estacionariedade do resíduo da equação co-integrante de longo prazo.

Os resultados da análise de causalidade bivariada são reportados na Tabela 5. O mecanismo de correção de erros comparece significativamente em todas as equações dos modelos ADL, indicando a existência de co-integração entre cada par de variáveis. O teste de causalidade inclui o MCE na significância conjunta dos coeficientes defasados de cada modelo ADL, tornando-se 
o teste de causalidade de Engle-Granger. A seleção das defasagens nos modelos ADL se deu pelo critério de busca "geral para o específico" com base na significância estatística a 5\% do último coeficiente defasado pelo teste $t$. Quando não foi possível selecionar alguma defasagem por esse método, recorreu-se aos critérios de informação de Akaike e Schwarz.

\section{Tabela 5 - Teste de Causalidade de Engle-Granger}

\begin{tabular}{|c|c|c|c|c|c|}
\hline Hipótese Nula & Obs. & $\mathrm{F}$ & Valor-p & Causa & $A D L$ \\
\hline$\Delta i_{t}$ não Granger-Causa $\Delta d_{t}$ & 117 & $2,03^{* *}$ & 0,0212 & SIM & $\operatorname{ADL}(14,14)$ \\
\hline$\Delta d_{t}$ não Granger-Causa $\Delta i_{t}$ & 117 & 0,91 & 0,5488 & NÃO & $\operatorname{ADL}(14,14)$ \\
\hline$\Delta s p_{t}$ não Granger-Causa $\Delta d_{t}$ & 123 & $5,59^{*}$ & 0,0000 & SIM & $\operatorname{ADL}(8,8)$ \\
\hline$\Delta d_{t}$ não Granger-Causa $\Delta s p_{t}$ & 123 & 1,74 & 0,0876 & NÃO & $\operatorname{ADL}(8,8)$ \\
\hline$\Delta e m b i_{t}$ não Granger-Causa $\Delta d_{t}$ & 130 & $4,55^{\star \star}$ & 0,0123 & SIM & $\operatorname{ADL}(1,1)$ \\
\hline$\Delta d_{t}$ não Granger-Causa $\Delta e m b i_{t}$ & 130 & $3,36^{\star *}$ & 0,0375 & SIM & $\operatorname{ADL}(1,1)$ \\
\hline$\Delta z_{t}$ não Granger-Causa $\Delta d_{t}$ & 129 & $2,76^{\star *}$ & 0,0448 & SIM & $\operatorname{ADL}(2,2)$ \\
\hline$\Delta d_{t}$ não Granger-Causa $\Delta z_{t}$ & 129 & $17,37^{*}$ & 0,0000 & SIM & $\operatorname{ADL}(2,2)$ \\
\hline$\Delta z_{t}$ não Granger-Causa $\Delta e m b i_{t}$ & 129 & 1,70 & 0,1853 & NÃO & $\operatorname{ADL}(2,1)$ \\
\hline$\Delta e m b i_{t}$ não Granger-Causa $\Delta z_{t}$ & 126 & $3,96^{*}$ & 0,0012 & SIM & $\operatorname{ADL}(2,5)$ \\
\hline$\Delta i_{t}$ não Granger-Causa $\Delta e m b i_{t}$ & 129 & $3,70^{\star *}$ & 0,0136 & SIM & $\operatorname{ADL}(2,2)$ \\
\hline$\Delta e m b i_{t}$ não Granger-Causa $\Delta i_{t}$ & 124 & $10,22^{*}$ & 0,0000 & SIM & $\operatorname{ADL}(7,2)$ \\
\hline$\Delta s p_{t}$ não Granger-Causa $\Delta e m b i_{t}$ & 126 & $4,73^{*}$ & 0,0002 & SIM & $\operatorname{ADL}(2,5)$ \\
\hline$\Delta e m b i_{t}$ não Granger-Causa $\Delta s p_{t}$ & 123 & $5,18^{*}$ & 0,0000 & SIM & $\operatorname{ADL}(2,8)$ \\
\hline$\Delta i_{t}$ não Granger-Causa $\Delta z_{t}$ & 127 & 1,73 & 0,1317 & NÃO & $\operatorname{ADL}(2,4)$ \\
\hline$\Delta z_{t}$ não Granger-Causa $\Delta i_{t}$ & 129 & $5,02^{*}$ & 0,0025 & SIM & $\operatorname{ADL}(1,2)$ \\
\hline$\Delta s p_{t}$ não Granger-Causa $\Delta z_{t}$ & 120 & $3,98^{\star}$ & 0,0000 & SIM & $\operatorname{ADL}(2,11)$ \\
\hline$\Delta z_{t}$ não Granger-Causa $\Delta s p_{t}$ & 129 & $5,01^{*}$ & 0,0081 & SIM & $\operatorname{ADL}(2,1)$ \\
\hline$\Delta s p_{t}$ não Granger-Causa $\Delta i_{t}$ & 119 & $4,79^{*}$ & 0,0000 & SIM & $\operatorname{ADL}(1,12)$ \\
\hline$\Delta i_{t}$ não Granger-Causa $\Delta s p_{t}$ & 123 & $4,95^{\star}$ & 0,0000 & SIM & $\operatorname{ADL}(2,8)$ \\
\hline
\end{tabular}

Nota: * $\operatorname{significância~a~1\% ;~**~significância~a~5\% ;~***~significância~a~10\% .~Na~escolha~do~número~ótimo~de~defasagens,~}$ partiu-se de 14 defasagens. Os modelos foram estimados com a inclusão de variável dummy para fevereiro de 1999. "Obs." é o n. de observações.

Da mesma forma que na Tabela 4, algumas relações de bicausalidade foram observadas. Contudo, o resultado fundamental que direcionou a conclusão para existência de um regime de dominância monetária se manteve.

A relação superávit primário/PIB Granger-causa unidirecionalmente a relação dívida/PIB ao nível de significância de $1 \%$. Isto confirma o resultado da análise multivariada, segundo o qual a trajetória da relação dívida/PIB é afetada por variações na geração de superávits primários. Ou seja, melhorias na estabilização da relação dívida/PIB podem ser obtidas por meio da 
geração de superávits primários. Além de expressar ganhos de credibilidade da política fiscal, esse resultado caracteriza um país sob regime de dominância monetária segundo definição de Sargent e Wallace (1981), pois a política fiscal garante a sustentabilidade da dívida pública.

A taxa Selic também Granger-causa unidirecionalmente a relação dívida/PIB ao nível de significância de 5\%. Isto, também em conformidade com o teste de causalidade multivariado, sugere a existência de uma autoridade monetária autônoma, que é capaz de fixar a taxa Selic ao nível de sua escolha, expressando, portanto, ganhos de credibilidade da política monetária. Logo, a política monetária não é afetada pela dinâmica da dívida pública, o que também caracteriza um regime de dominância monetária.

O resultado anterior refuta o argumento de Blanchard (2004), segundo o qual a precedência temporal iria da dívida pública para o superávit primário. As relações de bicausalidade reportadas na Tabela 5 também contradizem o ciclo vicioso descrito por Blanchard (2004), que implicitamente pressupõe exogeneidade entre as variáveis que não encontrou respaldo empírico.

Por exemplo, há bicausalidade, no sentido de Granger, entre a taxa Selic e o spread do EMBI ao nível de significância de 1\%, confirmando o resultado da análise multivariada. Por um lado, se variações na taxa Selic precedem temporalmente variações no spread do EMBI, então melhorias na avaliação externa da economia brasileira podem ser obtidas via política monetária. Ou seja, uma decisão de reduzir a taxa de juros levaria a uma queda do risco país, melhorando o status dos títulos nacionais e a avaliação externa da economia brasileira no mercado financeiro internacional. Por outro lado, se as variações no spread do EMBI precedem temporalmente variações na taxa Selic, pode-se argumentar que a taxa nominal de juros doméstica é elevada porque o País é arriscado ou porque ainda não conquistou sua credibilidade na política monetária.

Observa-se, também, relação de bicausalidade entre o spread do EMBI e a relação dívida/ PIB, ao nível de significância de 5\%, confirmando o resultado da análise multivariada. A relação de causalidade de Granger unidirecional do spread do EMBI para a relação dívida/PIB mostra que o risco país tem importância para se entender as variações no grau de endividamento público devido ao efeito que o risco país exerce sobre a avaliação externa da economia brasileira e na disponibilidade de recursos a serem destinados para a compra de títulos públicos internos e externos. Portanto, melhorias na avaliação externa do País podem contribuir para a estabilização da relação dívida/PIB, porque é grande a probabilidade de que a história passada do spread do EMBI afete a trajetória da relação dívida/PIB. Esse resultado está de acordo com o estudo de Reinhart e Rogoff (2004) que, ao analisarem dados históricos de incidência de default da dívida pública externa para países da Europa e da América Latina, inclusive o Brasil, verificaram que a principal explicação para o paradoxo de Lucas (1990) está no fato de que os países que não pagaram suas dívidas possuem relativa dificuldade temporal em obter empréstimos do resto do mundo. O histórico de default refletido no EMBI Granger-causa a relação dívida/ PIB. 
Por outro lado, a relação de causalidade de Granger unidirecional da relação dívida/PIB para o spread do EMBI evidencia que o grau de endividamento público afeta a avaliação externa da economia brasileira.

Assim, as análises de causalidade de Granger apresentadas nas Tabelas 4 e 5 permitem concluir que o Brasil encontra-se sob um regime de dominância monetária, conforme definição proposta por Sargent e Wallace (1981). Além disso, a transmissão entre variáveis fiscais e monetárias assumida por Blanchard (2004) não possui respaldo empírico.

\section{CONCLUSÕES}

Este estudo buscou verificar a existência de um regime de dominância fiscal ou monetária na economia brasileira no período pós-Plano Real por meio do teste de causalidade de Granger. Utilizaram-se duas metodologias distintas, uma baseada na análise multivariada por meio da estimação de um VEC e outra envolvendo a estimação de modelos ADL para variáveis cointegradas.

Os resultados das análises de causalidade bivariada e multivariada indicaram que a taxa Selic Granger-causa unidirecionalmente a relação dívida/PIB. Isto fortalece o argumento da existência de uma autoridade monetária autônoma, capaz de fixar a taxa Selic ao nível de sua escolha, o que expressa ganhos de credibilidade da política monetária. Em outras palavras, a política monetária não é afetada pela dinâmica da dívida pública, caracterizando um regime de dominância monetária.

A relação superávit primário/PIB também Granger-causa unidirecionalmente a relação dívida/PIB, revelando que a trajetória da relação dívida/PIB é afetada por variações na geração de superávits primários. Esse resultado é robusto nas análises de causalidade bivariada e multivariada. Logo, melhorias na estabilização da relação dívida/PIB podem ser obtidas por meio da geração de superávits primários, expressando ganhos de credibilidade da política fiscal, contradizendo o argumento de Blanchard (2004) de existência de um regime de dominância fiscal.

Entretanto, se existem ganhos de credibilidade das políticas fiscal e monetária, por que a avaliação externa ainda exerce influência na economia brasileira? A resposta reside no fato de que a credibilidade adquirida ainda é frágil. Conforme destacam Reinhart e Rogoff (2004), os mercados ainda levam em consideração o histórico de default do Brasil, ou seja, a memória dos mercados sobre a moratória brasileira da década de 1980 ainda está presente. Isto determina a influência interna da avaliação externa do País.

As análises de causalidade multivariada e bivariada não corroboram a transmissão entre variáveis fiscais e monetárias assumidas a priori por Blanchard (2004). Isto torna os resultados de seu modelo insuficientes para classificarem o Brasil como um País sob regime de dominân- 
cia fiscal. Além disso, os resultados aqui apresentados vão de encontro às conclusões de Tanner e Ramos (2002). Contudo, utilizando-se uma metodologia distinta, corrobora-se a evidência de Fialho e Portugal (2005). A análise de causalidade de Granger mostrou que a economia brasileira encontra-se em um regime de dominância monetária.

\section{REFERÊNCIAS}

BLANCHARD, O. Fiscal dominance and inflation targeting: lesson from Brazil. NBER WP, n. 10389, 2004.

CAMPBELL, J. Y.; PERRON, P. Pitfalls and opportunities: what macroeconomics should know about unit roots. In: BLANCHARD, O. J.; FISCHER, S. (Org.). NBER Macroeconomics Annual, n. 6. Cambridge: MIT Press, 1991.

COCHRANE, J. H. Long-term debt and optimal policy in the fiscal theory of the price level. Econometrica, v. 69, n. 1, p. 69-116, 2001.

DICKEY, D. A.; FULLER, W.A. Distribution of the estimators for autoregressive time series with unit root. Journal of the American Statistical Association, v. 74, n. 336, 1979. $4, \overline{19} 81$.

Likelihood ratio statistics for auto-regressive time series with unit root. Econometrica, v. 49, n.

ELLIOT, G.; ROTHENBERG, T. J.; STOCK, J. H. Efficient tests for an autoregressive unit root. Econometrica, v. 64, n. 4, p. 813-836, 1996.

ENGLE, R. F.; GRANGER, C. W. J. Co-integration and error correction: representation, estimation and testing. Econometrica, v. 55, n. 2, p. 251-276, 1987.

FIALHO, M. L.; PORTUGAL, M. S. Monetary and fiscal policy interactions in Brazil: an application to the fiscal theory of the price level. Estudos Econômicos, São Paulo, v. 35, n. 4, p. 657-685, 2005.

GRANGER, C. W. J. Investigating causal relations by econometric models and cross-spectral models. Econometrica, v. 34, p. 541-551, 1969.

JOHANSEN, S.; JUSELIUS, K. Maximum likelihood estimation and inference on co-integration with applications for the demand for money. Oxford Bulletin of Economics and Statistics, v. 52, p. 169-210, 1990.

JOHANSEN, S. Estimation and hypothesis test of cointegration vectors in Gaussian vector autoregressive models. Econometrica, v. 59, p. 1551-1580, 1991.

LUCAS, R. Why doesn't capital flow from rich to poor countries? American Economic Review, v. 80, p. 92-96, 1990.

NG, S.; PERRON, P. Lag length selection and the construction of unit root tests with good size and power. Econometrica, v. 69, n. 6, 2001.

PERRON, P. The great crash, the oil price shock, and the unit root hypothesis. Econometrica, v. 57, n. 6. p. 1361-1401, 1989.

PHILLIPS, P. C. B. Time series regression with unit roots. Econometrica, v. 55, p. 277-302, 1987. $198 \overline{8}$.

.; PERRON, P. Testing for unit root in time series regression. Biometrika, v. 75, n. 2, p. 335-346, 
REINHART, C. M.; ROGOFF, K. S. Serial default and the "paradox" of rich to poor capital flows. American Economic Review, v. 94, n. 2, 2004.

SAID, S.; DICKEY, D. A. Testing for unit roots in autoregressive-moving average models of unknown order. Biometrika, v. 71, 1984.

SARGENT, T. J.; WALLACE, N. Some unpleasant monetarist arithmetic. Quarterly Review, Federal Reserve Bank of Minneapolis, v. 5, n. 3, p. 1-17, 1981.

SIMS, C. A. A simple model for study of the price level and the interaction of monetary and fiscal policy. Economic Theory, v. 4, n. 3, p. 381-399, 1994.

TANNER, E.; RAMOS, A. M. Fiscal sustainability and monetary versus fiscal dominance: evidence from Brazil, 1991-2000. Internacional Monetary Fund, Washington, DC, 2002. (WP n. 5).

WOODFORD, M. Monetary policy and price level determinacy in a cash-in-advance economy. Economic Theory, v. 4, n. 3, p. 345-380, 1994.

. Price level determinacy without control of a monetary aggregate. Carnegie Rochester Conference Series on Public Policy, v. 43, p. 1-46, 1995. 728,2001

Fiscal requirements for price stability. Journal of Money, Credit and Banking, v. 33, n. 3, p. 669- 
